\title{
Evaluation and Comparison of Episodic Memory in Elderly People: The Effect of Strategies Education on Memory Reinforcement
}

5. Mousavi (Shokoufeh Mousavi)', A. Motamedi (Abdollah Motamedi)², E. Honejani (Esmail Honejani)33, S. M. Zadehhossein (Sayed Mohsen Zadehhossein)4, N. Mirjahanian (Nooshin Mirjahanian) ${ }^{5}$

Original Article

1,2,4 Department of Psychology, Payame Noor University

3 Master of Science in Psychology, Department of Education, Isfahan, Iran

5 Department of Psychology, Khomeinishahr Branch, Islamic Azad University, Khomeinishahr/Isfahan, Iran

\section{E-mail address:}

rooyamousavi@gmail.com

\section{Reprint address:}

Shokoufeh Mousavi

Department of Psychology

Payame Noor University

PO BOX 19395-3697

Tehran, Iran

Suource: Clinical Social Work and Health Intervention Pages: $59-66$
Volume: 8

Issue: 2

\section{Reviewers:}

Katarina Zoller

UNHCR Refugee Camp

Veria, Greece

Alexandra Topolska

St. Elisabeth University of Health and Social Sciences, Bratislava, Slovakia Missionary in Yemen

\section{Key words:}

Episodic memory, Semantic memory, Verbal memory, Practical memory, Elderly, Loss of memory, Memory reinforcement strategies.

\section{Publisher:}

International Society of Applied Preventive Medicine i-gap

CSWHI 2017; 8(2): 59 - 66; DOI 10.22359/cswhi_8_2_11 @ 2017 Clinical Social Work and Health Intervention 


\section{Abstract:}

The current study obtains a framework to compare the episodic memory among two groups of elderly people with and without training in strategies for enhanced memory. A total of 24 elderly people were selected in a convenience sampling method and then divided into two groups. One group was trained with memory reinforcement strategies and the other group was not. Verbal task and subject performed task were applied for assessment of episodic memory. The memory was evaluated by free recall test. The result of a T-test and ANOVA showed there was a significant difference between two groups in verbal and subject performed tasks. The group trained in memory reinforcement strategies showed higher performance than the untrained one. Applying memory reinforcement strategies in learning can reduce long term memory loss in elderly people. Memory loss in the elderly is due to their not using encoded learning material with the richness and accuracy of the younger ones.

\section{Introduction}

In recent years, the issue of the minds of people of old age has attracted the attention throughout the world as a basic issue especially when 1999 was named the World Year of Old Age (Motamedi 2005). The issue of memory and its improvement is crucially important at this time. Old age weakens the neural system and leads to defect in cognitive functions including memory, learning and language-related and attention-related abilities (Ravona 2010). The results of a study done in Iran have shown that 53.8\% of old people being studied have had a mild cognitive disorder and $44 \%$ of them have had an average cognitive disorder (Eftekhar Ardebili et al. 2012). Memory is vital for human beings and other living creatures. Practically, all of the everyday life activities of human beings such as speaking, perceiving or understanding, reading and even sociability depend on information learnt and saved by individuals about their surrounding environment (Saed 2010).

In the history of Cognitive Psychology, various definitions of memory have been presented. Schacter (1996), has defined memory as the ability of keeping (saving) and rereading information, personal experiences and procedures (skills and habits). Also, memory includes processes through which human beings or other creatures encode, accumulate and recover information. Memory is one's ability to save information and experiences and using them in future interactions with the environment (Saed, 2010).

In the classifications of memory which have been presented, Tulving (1972) has divided long-term memory to two kinds of memory which are semantic and episodic. He believes that there are two information processing systems in the long-term memory of human beings.

In episodic memory, information or incidents have a certain time and place and there are time and spatial relationships between all types of information and these types of information are experienced personal1y. An example would be names of friends who attended a party last night. Semantic memory includes public information such as language, knowledge, vocabulary, laws, formulas and concepts which are mutual in 
human beings. This information does not have much time and spatial features and they are not exposed to change, evolution or oblivion (Karami Noori, 2004).

One of the methods which can lead to a change in the process of memory and to an increase in its functions is using memory reinforcement strategies. In the domain of Cognitive Psychology, the effectiveness of various strategies for the reinforcement of memory have been tested and proved. Some of these strategies are:

- creation of focus and attention;

- organization of information on one's mind;

- using retentive tools or props for remembering information;

- developing and reviewing information in one's mind;

- establishing a relationship between new and old information;

- imagining concepts in one's mind;

- paying more attention to complex concepts (Mahmoud Pour, 2008).

Many Studies show that unlike what was believed in the past, aging of various parts of the body including the brain is not affected by the genes, but the elements associated with the lifestyle which are changeable have just as much of an effect on aging (Rowe \& Kahn, 1999). Therefore, reduction of function in old people is not unavoidable and elderlies can maintain their mental and physical functions (Beckett et al. 1996; Motamedi 2005). Using memory reinforcement strategies is one of the methodologies which lead to the maintenance of mental functioning in the elderly.

Although much research has been done on the elderly and their mental processes in this period, the number of the researches that investigate utilization of memory reinforcement strategies to improve various types of memory and especially episodic memory is very low. The results of various research findings such as Burke \& Light (1982), Light (1991, 1992), Craik \& Jennings (1992), and Allen et al. (2002, 2005), have shown that old age reduces the function of episodic memory and these individuals are weaker in recovering information in episodic memory assignments than young people.

In a study done on 60-year-old and older elderlies in Shahrekord analyzed intellectual function in old age and investigated its role in successful old age, showed that intellectual function of elderlies is not significantly different in various age groups and intellectual function of elderlies who are older than 60-years does not reduce results of the regression analysis in this research showed that the intellectual function of successful elderlies has been significantly better than unsuccessful ones (Motamedi \& Ejeyi 2003).

Nejati (2012), in a research, investigated the relationship between active memory and verbal fluency in the elderlies and showed that there is a significant and positive correlation between verbal fluency and the direct section of spans of numbers and also between the indirect section of the number spans test and phonological verbal fluency test.

Thus, given the daily increasing importance of old age and the developing processes of this population, considering and recognizing the ways that can reinforce their memory and mental functions play important roles in the health of the elderly helping to maintain the independence and living quality of this maturing group.

The present study aims to compare episodic memory in old people who use memory reinforcement strategies with old people who do not use these strategies. The hypotheses of this research are: 
1. Elderly people who use memory reinforcement strategies are different in verbal memory than elderly people who don't use memory reinforcement strategies.

2. Elderly people who use memory reinforcement strategies are different in practical memory than the elderly people who don't use memory reinforcement strategies.

In this study which has been done through the Comparative Causal Method, 24 persons older than 60 years were randomly selected. Then, they were randomly divided into two groups:

- memory reinforcement strategies were taught to one group and they used it;

- the second group were tested without being taught memory reinforcement strategies;

- then, each group received practical and verbal memory assignments.

\section{Episodic Memory Test}

In this study, the episodic memory test included 32 short imperative sentences selected based on the study of Karami Noori and Moosavi (2008). 32 sentences were divided into two 16-sentence lists and each of them were used for testing verbal and practical memory. In order to do the episodic memory tests, after presenting the instructions and some examples to the samples, the person is asked to do some assignments and after the list is finished, in order to create a distance between the stages of encoding and remembering, the semantic memory test was done. The reason for the creation of this distance was for the long-term learning memory to be formed for the testees.

All of the tests were taken individually and the time it took each individual to take the test was about 120 minutes. It was attempted to provide a peaceful, calm and noiseless place without traffic for the tests to be taken.

In order for the testees not to use practical strategies in verbal encoding, the verbal list was presented to the testees in the beginning. In the first group and for the presentation of the verbal, the sentences are read to the testees and they must repeat them and memorize them. After presenting a verbal list and creating an approximately 30-minute time distance (at that time the testees received the semantic memory test), the free remembering test was done during which a white sheet is given to them and they are asked to write down any number of sentences that they remember in their order of preference. Then, the second list of the sentences is given to them and they must do the assignment with the tools given to them simultaneously after hearing the sentences. In the physical assignment, sentences are presented visually and for a longer duration. After finishing the list and a 30-minute time difference (receiving semantic memory tests), the testees again receive a sheet associated with free remembering. Here, the score is given to the testee if they remember the same exact sentence as the text.

\section{Results}

Mean and standard deviation of presenting tasks in two elderly groups are shown in Table 1. T-test results between the two groups are shown in Table 2 and $\mathbf{3}$. According to the results there was a significant difference between the two groups of elderly people by using the strategy and without using the strategy; so the elderly who used enhance memory strategies, had a better performance in verbal and practical task compared to elderly people who did not use these strategies. ANOVA Analysis was also performed to ensure which group showed a significant difference. ANOVA Summary of the two verbal and practical tasks are shown in Tables $\mathbf{4}$ and $\mathbf{5}$. 
Table 1: Descriptive characteristics (mean and standard deviation) of providing tasks

\begin{tabular}{|l|l|l|l|}
\hline Types of Memory & Number & Mean & SD \\
\hline Verbal Memory & 12 & 2.12 & 1.59 \\
\hline Without Strategy & 12 & 6.04 & 1.25 \\
\hline With Strategy & 12 & 4.25 & 2.04 \\
\hline Practical Memory & 12 & 10.75 & 0.78 \\
\hline Without Strategy &
\end{tabular}

Table 2: Results of $\mathrm{t}$ test between the two groups in the verbal task

\begin{tabular}{|l|l|l|c|}
\hline Index & $\mathrm{T}$ & dif & Two-tailed significance \\
\hline Verbal & -6.686 & 22 & 0.0001 \\
\hline
\end{tabular}

Table 3: Results of t test between the two groups in the practical task

\begin{tabular}{|l|l|l|c|}
\hline Index & $\mathrm{T}$ & dif & Two-tailed significance \\
\hline Verbal & -10.29 & 22 & 0.0001 \\
\hline
\end{tabular}

Table 4: Summary of ANOVA for the scores of testee in verbal task

\begin{tabular}{|l|c|c|c|l|l|}
\hline Sources of changes & SS & dif & MS & F & Sig \\
\hline Between group & 92.04 & 1 & 92.42 & - & - \\
\hline Intergroup & 45.292 & 22 & 2.059 & 44.708 & 0.0001 \\
\hline Total & 137.333 & 23 & - & - & - \\
\hline
\end{tabular}

Table 5: Summary of ANOVA for the scores of testee in practical task

\begin{tabular}{|l|c|c|c|c|c|}
\hline Sources of changes & SS & dif & MS & F & Sig \\
\hline Between group & 253.500 & 1 & 253.500 & - & - \\
\hline Intergroup & 52.625 & 22 & 2.392 & 105.976 & 0.0001 \\
\hline Total & 306.125 & 23 & - & - & - \\
\hline
\end{tabular}

Results of the present study showed that verbal memory means in the group that used memory enhancement strategies and the group that did not use of memory enhancement strategies was 6.04 and 2.15 respectively (Table 1), in which the difference between the two groups was significant $(\mathrm{t}$ $=-6.686$ and sig $=0.001$ ) (Table 2). Also, the results showed that the practical memory mean in both groups was 10.75 and 4.25 in which the difference between the two groups was significant $(\mathrm{t}=-10.294$ and $\mathrm{sig}$ $=0.001)($ Table 3).

\section{Discussion and Conclusion}

The elderly population over the past few years and the increase of hope for life has led to the increase of the attention paid to the quality of life in the period of old age. It seems that presentation of a successful old age and drawing the coordinates and features of successful old age present a kind 
of qualitative approach to the period of old age. Shaie (1990), considers successful old age as maximizing cognitive action (Motamedi \& Ejeyi 2003), and practical and verbal memory belongs to this function.

Old age phenomenon includes biological changes along with reduction in the abilities of living and the compatible response of the person against sudden changes (Gorman 2000). Old age is a sensitive period and it is necessary to pay attention to its needs and issues. There are various methods for maintaining mental functions in this period. The purpose of this research is to review and compare the episodic memory of elderlies who use memory reinforcement strategies and those who don't.

Generally, the results indicated that the mean of scores in both verbal and practical memory is higher in the group the member of which use memory reinforcement strategies. In other words, in this study, it has been shown that the old people who use memory reinforcement strategies are at a higher level than those who don't use memory reinforcement strategies in terms of practical and verbal memory. In the field of change of episodic memory in old age, these results comply with the findings of Burke \& Light (1982), Light (1991, 1992), Craik \& Jennings (1992) \& Allen et al. (2002, 2005), Motamedi \& Ejeyi (2003), and Nejati (2012).

Navabi Nejad (2005) expresses that memory strategies and mechanisms are reduced as the person gets older but the studies have shown that content of memory, i.e. saved knowledge, is usually increased. Also, various factors such as practical and mental factors, food, alcohol consumption and motivation affects the memory function in old age. Johnson et al. (1988), have identified various factors which affect the function of individuals' episodic memory. One of the factors that can be classified as mental function is the utilization of memory reinforcement strategies. Meaningful processing; active involvement; generation effect; repetition effect are among these concepts, each of which is close to the aforementioned reinforcement strategies.

Usually, information or episodes meaningful processing creates a better recovering process than processing that only focuses on surface features. Also, active involvement in the learning process such as information generation not only makes their rereading better, but also their recovering and remembering and this is called the generation effect. In addition, the episodes that are repeated more than once are remembered and recovered better than those that are repeated just once. Semantic development methods; usage of tools that help remembering; development and overview of the aforementioned information can be seen in memory reinforcement strategies, like the aforementioned concepts, helps the person improve the function of episodic memory, or practical and verbal memory. Repetition of the information in one's mind, using assistive tools and semantic development of information lead to a better maintenance of words in verbal memory and makes memory more successful in recovering them. In addition, using these strategies leads to a stronger and more reliable formation of procedures in memory and their recovery easier.

In addition, one of the most important memory reinforcement strategies is focusing and concentration. Focusing and concentration is one of the most important principles in converting and transferring information from sensual memory to shortterm memory and from short-term memory to long-term memory. In other words, it has an important role in encoding and 
recovering information. The principle of transfer appropriate processing, as one of the basic ideas in this field, expresses how information processing are associated with each other at the stages of encoding and recovering and their compatibility with one another causes a better return for remembering information. In terms of episodic memory, focusing and concentrating on information and events helps the encoding of the information in the memory and the achievement of a better recovery in time.

Of course, this study investigates the difference between elderlies who use memory reinforcement strategies and those who don't use it. Therefore, it is recommended to other researchers to review and compare these groups in various age populations and to consider and control variables such as social class or education.

\section{References}

1. ALLEN PA, KAUT KP, LORD RG, HALL RJ, GRABBE JW, BOWIE T (2005). An emotional mediation theory of differential age effects in episodic and semantic memories. Experimental Aging Research: 31: 355-391.

2. ALLEN PA, SLIWINSKI M, BOWIE T (2002). Differential age effects in semantic and episodic memory, Part II: slope and intercept analyses. Experimental Aging Research. 28:111-142.

3. BURKE DM, LIGHT LL (1982). Memory and aging: the role of retrieval processes. Psychical Bull. 90: 513-546.

4. CRAIK FIM, JENNINGS JM (1992). Human memory. In: Craik FIM. Salthouse TA (Eds.), The Handbook of Aging and Cognition. Erlbaum, Hillsdale, NJ, pp. 51-110.

5. EFTEKHAR ARDEBILI H, KHATI F, BATEBI A, SHOJAEIZADEH D, YAZDANI CHARATI J (2012). Evaluate the frequency cognitive impairment, functional and associated factors among the elderly. Mazandaran University of Medical Sciences. 22(96): 115-125.

6. GORMAN M (2000). Development and the right of older people In: Randel J et al. (eds). The aging and development report: poverty, independence and the world's older people. London: Earths can publications ltd. P.3-21.

7. JOHNSON MK, FOLEY MA, SUENGAS AG, RAYE CL (1988). Phenomenal characteristic of memories for perceived and imagined autobiographical events. Journal of Experimental Psychology. 117: 371-376.

8. KARAMI NOORI R (2004). Psychology of memory and learning, cognitive approach. First Edition. Publisher: Samt. Tehran, Iran.

9. KARAMI NOORI R, MOUSAVI S (2008). Compare variety of episodic and semantic memory in deaf and hearing students. Recent Cognitive Science. 10 (4): 83-93.

10. LIGHT LL (1991). Memory and aging: four hypotheses in search of data. Annual Review Psychology. 42: 333-376.

11. LIGHT LL (1992). The organization of memory in old age. In: Craik FIM, Salthouse TA (Eds.) The Handbook of Aging and Cognition. Erlbaum, Hillsdale, NJ, pp. 111-167.

12. MAHMOUD POUR A (2007). Methods and strategies for memory enhancement. Eighth Edition, Publisher: School. Tehran, Iran.

13. MOTAMEDI A (2005). The role of life events on successful aging. Journal of $\mathrm{Hu}-$ manities and Social Sciences. Shiraz University. 24(4): 189-203.

14. MOTAMEDI A, EJEYI J (2003). Analysis of the nature of intelligence in old age and its relationship with successful aging. Journal of Psychology. 28: 398-421.

15. NAVABI NEJAD N (2005). Memory and its impact on performance of elderly. Journal of Medicine and Rehabilitation, 1 (1): 1218.

16. NEJATI V (2012). The relationship between active memory and verbal fluency in the 
elderly. Research in Rehabilitation Sciences. 3: 412-418.

17. RAVONA R (2010). Rate of cognitive decline is not affected by age in elderly individuals residing in nursing and assisted living facilities. Alzheimer's \& Dementia, the Journal of the Alzheimer's Association. 6(4): S449.
18. ROWE JW, KAHN RL (1999). The Future of Aging. Contemporary - Longterm Cave, New York: 22(2).

19. SAED A (2010). Psychological and biological basis of memory. First Edition. Publisher: Arjmand. Tehran, Iran.

20. SCHACTER DL (1996). Searching for memory- the brain, the mind, and the past, New York: Basic Books. 